\title{
Investigating Characteristics of Sensitive Galvanometer by Isogonic Method and SPSS
}

\author{
Kaihua YUE, Xinchun WANG, Qing YE \& Man CHENG \\ School of Physics and Electronics, Chuxiong Normal University, Chuxiong 675000, China
}

ABSTRACT: By making use of isogonic method and combining with SPSS, this paper attempts to measure the sensitivity and internal resistance of a sensitive galvanometer, evaluate experimental data and its uncertainty with confidence probability of $95 \%$. This paper introduces experimental circuit and principle, and gets reliable and reasonable results.

KEYWORD: Isogonic Method; Sensitive Galvanometer; SPSS; Sensitivity; Internal Resistance; Uncertainty

\section{INTRODUCTION}

Sensitive galvanometer is a kind of magnetoelectric ammeter with a high sensitivity. It can be used to measure micro current, low voltage, or used as a galvanometer directly. Investigating the characteristics of sensitive galvanometer is an important experiment of college physics [3]. According to reference [1], [4], [7] we know that, isogonic method is much better than half deviation method when measuring sensitivity and internal resistance of a sensitive galvanometer. So the isogonic method is used in this paper. In addition, uncertainty estimation (confidence probability of $95 \%$ ) is used to evaluate experimental data. Further more, by use of the curve estimation function of SPSS [6], error caused by instrument or human factors is reduced significantly.

\section{EXPERIMENTAL CIRCUIT AND CALIBRATION}

The experimental device is demonstrated in Figure1; it mainly includes a sensitive galvanometer, a regulated power supply, two resistance boxes, a standard resistor, a voltmeter, a rheostat and two switches (one of the two switches is a reversing switch).

The experimental device is shown in Figure 2. There are two voltage dividers in the circuit. The first voltage divider is formed by rheostat $R_{0}$, which can change the voltage $U$ from 0 to $E$. The voltage $U$ is indicated by a voltmeter. The second voltage divider consists of standard resistor $R_{1}$ and resistance box $R_{2}$. If $R_{1}<<R_{2}$, voltage $U_{1}$ across $R_{1}$ will be very small.

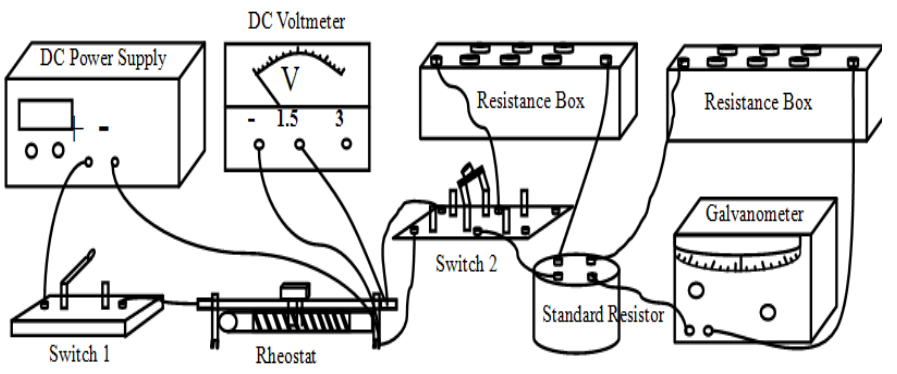

Figure 1. Experimental device

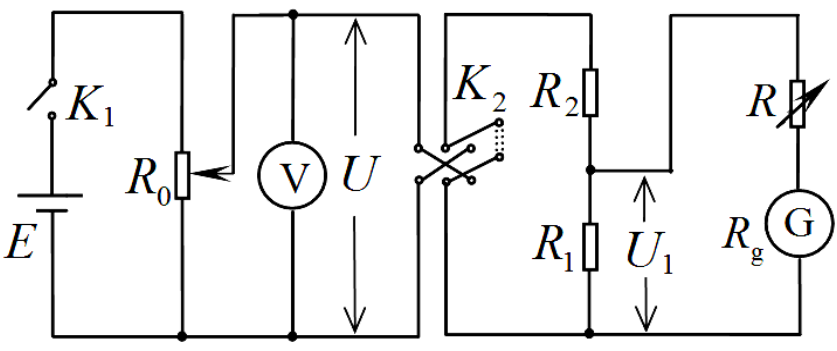

Figure 2.Experimental circuit

With $K_{1}, K_{2}$ open; calibrate the cursor of sensitive galvanometer coinciding with the zero point of the ruler. Fix $R_{1}=1 \Omega$ and $R_{2}=32 \mathrm{k} \Omega$, select cursor deflection number $N=40$ div . 


\section{EXPERIMENTAL PRINCIPLES}

\subsection{Measurement Principle}

In Figure 2, the equivalent resistance of $R_{1}$ and $\left(R+R_{\mathrm{g}}\right)$ is

$$
R_{1}^{\prime}=\frac{R_{1}\left(R+R_{\mathrm{g}}\right)}{R_{1}+R+R_{\mathrm{g}}}
$$

Voltage $U_{1}$ across $R_{1}$ and $\left(R+R_{\mathrm{g}}\right)$ is

$$
U_{1}=I_{g}\left(R+R_{\mathrm{g}}\right)=U \frac{R_{1}^{\prime}}{R_{1}^{\prime}+R_{2}}
$$

If $R_{1}<<R_{2}$, thus $R_{1}^{\prime}<<R_{2}$, equation (2) can be simplified as

$$
I_{g}\left(R+R_{\mathrm{g}}\right) \approx U \frac{R_{1}^{\prime}}{R_{2}}
$$

Substitute equation (1) into equation (3) gives

$$
R+R_{1}=\frac{R_{1}}{R_{2} I_{g}} U-R_{g}
$$

The sensitivity [9] is defined as

$$
S=\frac{N}{I_{g}}
$$

From equation (4) and equation (5) we get

$$
R+R_{1}=S \frac{R_{1}}{R_{2} N} U-R_{\mathrm{g}}
$$

Let $Z=\frac{R_{1}}{R_{2} N} U$ and $W=R+R_{1}$, then equation

(6) can be simplified as

$$
W=S Z-R_{\mathrm{g}}
$$

For convenience, we call $Z$ as comprehensive quantity, and call $W$ as resistance composite quantity.

In the experiment, close $K_{1}, K_{2}$, slide rheostat to make the voltage $U$ change from $0.6 \mathrm{~V}$ to $1.5 \mathrm{~V}$ gradually (take voltage step to be $0.1 \mathrm{~V}$ ). For each voltage, adjust resistance $R$ until the cursor deflection equals to $N$ (40div), now record $R$ as $R_{q}$; then reverse $K_{2}$, keep $U$ unchanged and adjust $R$ until the cursor deflection equals to $N$ (40div) again, record $R$ as $R_{h}$. The mean resistance is $R=\left(R_{q}+R_{h}\right) / 2$.

Apply curve estimation function of SPSS to the data; it is easy to find the relationship between the comprehensive quantity $Z$ and resistance composite quantity $W$, and calculate the sensitivity and internal resistance of the sensitive galvanometer.

\subsection{Uncertainty Analysis of Sensitivity}

For a direct measurement quantity $k$, its uncertainty can be divided into class $A$ and class $B$. The standard deviation [5] of a series data is

$$
u_{(\bar{k})}=\sqrt{\frac{\sum_{i=1}^{n}\left(k_{i}-\bar{k}\right)^{2}}{n(n-1)}}
$$

In class $A$, if the data is 10 or more, the uncertainty of $k$ obey $t$ distribution, when $p=0.95, t_{p}=2.26$ thus

$$
u_{A(\bar{k})}=2.26 u_{(\bar{k})}
$$

$\bar{k}$ in equations (8) and (9) stands for $\bar{R}, \bar{U}, \bar{R}_{1}$ or $\bar{R}_{2}$ respectively.

In class $B$, the instrument limit error $\Delta$ is subject to uniform distribution $C=\sqrt{3}$, when $p=0.95, k_{p}=1.96$, then

$$
u_{B(k)}=1.96 \frac{\Delta_{k}}{\sqrt{3}}
$$

where $k$ stands for $\bar{R}, \bar{U}, \bar{R}_{1}$ or $\bar{R}_{2}$ respectively.

The uncertainty of a direct measurement quantity $k$ is

$$
u_{(k)}=\sqrt{u_{A(\bar{k})}^{2}+u_{B(k)}^{2}}
$$

For indirect measurement quantity $y=f\left(k_{1}, k_{2}, \ldots, k_{i}, \ldots k_{m}\right)$, the standard uncertainty [2] is

$$
u_{c(y)}=\sqrt{\sum_{i=1}^{n}\left(\frac{\partial y}{\partial k}\right)^{2} u_{\left(k_{i}\right)}}
$$

The relative uncertainty is

$$
u_{r(y)}=\sqrt{\sum_{i=1}^{n}\left(\frac{\partial(\ln y)}{\partial k_{i}}\right)^{2} u_{\left(k_{i}\right)}}
$$

Consider equation (8), (13), the relative uncertainty of the sensitivity is

$$
u_{r\left(s_{i}\right)}=\sqrt{\left(\frac{u_{(W)}}{W}\right)^{2}+\left(\frac{u(z)}{z}\right)^{2}}
$$


4 EXPERIMENTAL DATA PROCESSING

\subsection{Measured Data and Processing}

Table 1. Parameters of sensitive galvanometer Ac15/6

\begin{tabular}{|c|c|c|c|}
\hline $\begin{array}{c}\text { Internal } \\
\text { resistance }[\Omega]\end{array}$ & $\begin{array}{c}\text { Critical external } \\
\text { resistance }\left[10^{2} \Omega\right]\end{array}$ & $\begin{array}{c}\text { Sensitivity } \\
{\left[10^{8} \text { div/A] }\right.}\end{array}$ & $\begin{array}{c}\text { Damp } \\
\text { time }[\mathrm{s}]\end{array}$ \\
\hline 38 & 4 & 4 & 4 \\
\hline
\end{tabular}

Table 2. Relationship between $U$ and $R$

\begin{tabular}{|c|c|c|c|c|}
\hline$i$ / Group & $U[\mathrm{~V}]$ & $R_{q}[\Omega]$ & $R_{h}[\Omega]$ & $R[\Omega]$ \\
\hline 1 & 0.598 & 149.50 & 148.10 & 148.80 \\
\hline 2 & 0.704 & 183.80 & 180.60 & 182.20 \\
\hline 3 & 0.797 & 214.50 & 212.10 & 213.30 \\
\hline 4 & 0.900 & 246.50 & 243.90 & 245.20 \\
\hline 5 & 0.999 & 279.70 & 275.80 & 277.75 \\
\hline 6 & 1.102 & 310.20 & 307.20 & 308.70 \\
\hline 7 & 1.199 & 338.60 & 341.80 & 340.20 \\
\hline 8 & 1.302 & 374.40 & 369.30 & 371.85 \\
\hline 9 & 1.401 & 402.60 & 402.20 & 402.40 \\
\hline 10 & 1.500 & 435.20 & 431.00 & 433.10 \\
\hline
\end{tabular}

where $R=\left(R_{q}+R_{h}\right) / 2, R_{1}=1 \Omega, R_{2}=32.00 \mathrm{k} \Omega$ ， $N=40 \operatorname{div}$ and $\Delta_{R}=0.1 \Omega$.

Table 3. Relationship between $W$ and $Z$

\begin{tabular}{|c|c|c|}
\hline$i$ /group & $W\left[10^{2} \Omega\right]$ & $Z\left[10^{-6} \mathrm{~V} /\right.$ div $]$ \\
\hline 1 & 1.4980 & 0.4671875 \\
\hline 2 & 1.8320 & 0.5500000 \\
\hline 3 & 2.1430 & 0.6226563 \\
\hline 4 & 2.4620 & 0.7031250 \\
\hline 5 & 2.7875 & 0.7804688 \\
\hline 6 & 3.0970 & 0.8609375 \\
\hline 7 & 3.4120 & 0.9367188 \\
\hline 8 & 3.7285 & 1.0171875 \\
\hline 9 & 4.0340 & 1.0945313 \\
\hline 10 & 4.3410 & 1.1718750 \\
\hline
\end{tabular}

\subsection{Using SPSS Software to Analyze the Calibration Curve}

Put experimental data in Table 3 into SPSS software, taking $Z$ as variable and $W$ as dependent variable. By use of the function of curve estimated of SPSS, we have the calibration curve equation

$$
W=4.035275236 \times 10^{8} Z-37.73173091
$$

The calibration curve is shown in Figure 3.
$W / 10^{2} \Omega$

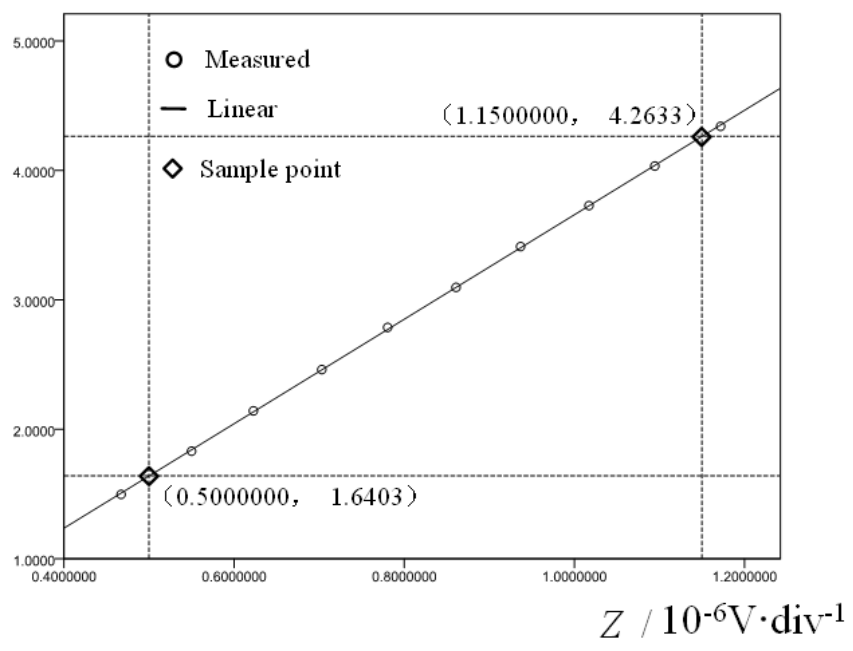

Figure 3.W-Z calibration curve obtained by SPSS

\subsection{Estimation of Uncertainty of Sensitivity}

According to Figure 3 , the calibration curve is a straight line. Take two points $\left(Z_{1}, W_{1}\right)$ and $\left(Z_{2}, W_{2}\right)$ from the straight line, we have

$$
\begin{aligned}
& S=\frac{W_{2}-W_{1}}{Z_{2}-Z_{1}} \\
& u\left(_{Z_{j}}\right)=\frac{u\left(W_{W_{j}}\right)}{4.035275236 \times 10^{8}}
\end{aligned}
$$

By equation (14), (16)

$$
u_{r(S)}=\frac{u_{(S)}}{S}=\sqrt{\left(\frac{u_{\left(W_{2}-W_{1}\right)}}{W_{2}-W_{1}}\right)^{2}+\left(\frac{u_{\left(Z_{2}-Z_{1}\right)}}{Z_{2}-Z_{1}}\right)^{2}}
$$

By Figure 3, combined with equation (16), (17), (18), we list experimental results in Table 4.

Table 4. Experimental results of the sensitive galvanometer

\begin{tabular}{|c|c|c|c|}
\hline $\begin{array}{c}u_{\left(W_{1}\right)}=u_{\left(W_{2}\right)} \\
{[\Omega]}\end{array}$ & $\begin{array}{c}u_{\left(z_{1}\right)}=u_{\left(z_{2}\right)} \\
{\left[10^{-10} \mathrm{~V} / \mathrm{div}\right]}\end{array}$ & $\begin{array}{c}S \\
{\left[10^{8} \mathrm{div} / \mathrm{A}\right]}\end{array}$ & $u_{r(S)}$ \\
\hline 0.16 & 4.0 & $4.035 \pm 0.035$ & $0.09 \%$ \\
\hline
\end{tabular}

\section{ANALYSIS AND CONCLUSION}

From the data in Table 3, by means of curve estimate function of SPSS, we get the calibration equation (15) and calibration curve (Figure 3). The calibration equation (15) is a linear equation, and calibration curve is a straight line. The intercept 37.73 of equation (15) is internal resistance of the sensitive galvanometer, which is very close to the value listed in Table 1 . The slope $4.03 \times 10^{8} \mathrm{div} / \mathrm{A}$ of equation (15) is the sensitivity of the sensitive galvanometer, which is in good agreement with $4 \times 10^{8} \mathrm{div} / \mathrm{A}$, the 
value listed in Table 1 . These show that the experimental results are very accurate.

In order to get linear equation (15), it is very important to choose proper parameters in the circuit. In the experiment, standard resistance is $1 \Omega$, one resistance box is $32 \mathrm{k} \Omega$, voltage changes from $0.6 \mathrm{~V}$ to $1.5 \mathrm{~V}$, operating the reversing switch. All these can reduce accidental error and system error, and make the measurement results more reliable and reasonable.

\section{REFERENCES}

[1] He Delin. Improvement on method of measuring sensitivity and internal resistance of sensitive galvanometer. University physics experiment. Vol.9 No.1: 27-30
[2] Liu Caiming. Estimation and expression of uncertainty in of University physics experiment. University physics. Vol.16 (8): 21-2

[3] Qi Jingang \& Gao Jiwei. Measurement of internal resistance and current constant of galvanometer. Journal of Changchun normal university (natural science edition). Vol.29 No.6: 27-29

[4] Shen Shizhang. Error analysis in measuring internal resistance of sensitive galvanometer. Physics experiment. Vol.4 No.2: 63-53

[5] Song Wenfu \& Zhu Li. Estimation and expression of uncertainty. University physics experiment. Vol.17 No.1:69-71

[6] Song Zhigang. 2008. SPSS 16.0 Guide to data analysis. Telecommunications Press

[7] Xu Shunsong. Reseach on characteristics of galvanometer. University physics. Vol.23 No.1:44-46

[8] Yang Shuwu \& Zhao Lizhu. 2007. General Physics experiment 2(Electromagnetism, version 4). Beijing: Advanced education press. 